\title{
Efficacy and safety of finerenone in patients with chronic kidney disease: a systematic review with meta-analysis and trial sequential analysis
}

\author{
Zhangning Fu ${ }^{1,2}$, Xiaodong Geng ${ }^{1,2}$, Kun Chi ${ }^{1,2}$, Chengcheng Song ${ }^{1,2}$, Di Wu ${ }^{2}$, Chao Liu ${ }^{2}$, Quan Hong ${ }^{2 \wedge}$ \\ ${ }^{1}$ Medical School of Chinese PLA, Beijing, China; ${ }^{2}$ Department of Nephrology, Chinese PLA General Hospital, Chinese PLA Institute of \\ Nephrology, State Key Laboratory of Kidney Diseases, National Clinical Research Center for Kidney Diseases, Beijing Key Laboratory of Kidney \\ Diseases, Beijing, China \\ Contributions: (I) Conception and design: Q Hong, C Liu, Z Fu; (II) Administrative support: Q Hong; (III) Provision of study materials or patients: \\ Z Fu, X Geng, K Chi, C Song; (IV) Collection and assembly of data: Z Fu, C Liu; (V) Data analysis and interpretation: Z Fu, C Liu, D Wu; (VI) \\ Manuscript writing: All authors; (VII) Final approval of manuscript: All authors. \\ Correspondence to: Quan Hong; Chao Liu. Department of Nephrology, Chinese PLA General Hospital, Chinese PLA Institute of Nephrology, State \\ Key Laboratory of Kidney Diseases, National Clinical Research Center for Kidney Diseases, Beijing Key Laboratory of Kidney Diseases, 28 Fuxing \\ Road, Beijing 100853, China. Email: redhq@163.com; chaoliu301@foxmail.com.
}

Background: The efficacy and safety of finerenone are unknown. Therefore, we performed this metaanalysis to investigate the efficacy and safety of finerenone in patients with chronic kidney disease (CKD).

Methods: We systematically searched for relevant studies in the PubMed, Embase and Cochrane Library databases from database inception until December 2020. We selected randomized controlled trials assessing finerenone treatment in patients with CKD.

Results: Four trials $(n=7,048)$ met the inclusion criteria. Compared with placebo, finerenone significantly reduced the urine albumin-to-creatinine ratio (UACR) in patients with CKD mean difference (MD), -0.30 [95\% confidence interval (CI), $-0.50,-0.11], \mathrm{P}<0.05\}$, and trial sequential analysis (TSA) confirmed this result. No significant difference was observed in eGFR in patients with CKD between the finerenone and placebo groups [MD, -0.90 (95\% CI, -3.84 to 2.04), P>0.05]. Overall, the frequency of adverse events was similar in the two groups [relative risk (RR), 1. 00 (95\% CI, 0.98, 1.02), P>0.05], and TSA confirmed this result. However, the finerenone group exhibited a lower risk of cardiovascular disorders and a higher risk of hyperkalemia than the placebo group [RR, 0.92 (95\% CI, 0.85, 0.99), P<0.05 and RR, 2.04 (95\% CI, 1.77, 2.34), $\mathrm{P}<0.00001$, respectively].

Discussion: This meta-analysis indicated that finerenone confers an important antiproteinuric effect on patients with $\mathrm{CKD}$ and reduces the risk of cardiovascular disorders in these patients. Finerenone may be a promising therapy option for patients with CKD.

Trial registration: PROSPERO registration number: CRD42021222404.

Keywords: Efficacy; safety; finerenone; chronic kidney disease (CKD); meta-analysis

Submitted Mar 31, 2021. Accepted for publication Jun 18, 2021.

doi: $10.21037 /$ apm-21-763

View this article at: https://dx.doi.org/10.21037/apm-21-763

^ ORCID: Chao Liu, 0000-0001-8815-3658; Quan Hong, 0000-0002-6839-7695. 


\section{Introduction}

Chronic kidney disease (CKD) has become a major public health problem due to its high incidence, poor prognosis and high cost of medical treatment (1). Type 2 diabetes mellitus (T2DM) is the most common cause of CKD and is now globally the single leading cause of end-stage renal disease (ESRD). When type 2 diabetes leads to CKD, the disease is usually referred to as diabetic kidney disease (DKD) or diabetic nephropathy (DN) (2). A substantial proportion of individuals with diabetes develop CKD as a result of their disease and/or other comorbidities, such as hypertension and nephron loss (3). Patients with chronic kidney disease are characterized by a progressive decline in health-related quality of life as well as a high risk of cardiovascular disease (CVD) (4). A variety of factors are involved in the pathophysiological development of CKD, including aldosterone. Aldosterone, a mineralocorticoid hormone, is a downstream target of the activation of the renin-angiotensin system (RAS) that may have an impact on human kidneys due to its potential ability to increase proteinuria and decrease renal function (5). In addition to its effects on the kidney, aldosterone also has the potential to exert effects on the heart. Aldosterone can affect nonepithelial cells such as cardiomyocytes, endothelial cells, vascular smooth muscle cells (VSMCs), mesangial cells, and podocytes via the mineralocorticoid receptor and subsequent genomic events, as well as through nongenomic pathways. Current studies have revealed that aldosterone causes inflammation in various ways, including stimulating the formation of reactive oxygen species (ROS), endothelial exocytosis and adhesion, leading to fibrosis and remodeling in the heart and kidney (6).

In this way, blockade of aldosterone using mineralocorticoid receptor antagonists (MRAs) might be an effective strategy to treat CKD. Several experimental and clinical studies have provided evidence on the moderate renoprotective effect of classic steroidal MRAs (spironolactone and eplerenone) (7-16). However, their use in CKD patients is limited due to its potential risks of hyperkalemia, erectile dysfunction and menstrual disorders (5).

Finerenone (BAY 94-8862) is currently the most advanced third-generation nonsteroidal MRI drug. Preclinical studies have shown that finerenone has a higher selectivity than spironolactone, a better affinity than eplerenone and a lower risk of hyperkalemia $(17,18)$. Researchers have investigated the efficacy and safety of finerenone in recent years. However, its clinical efficacy and safety are still unclear.
Therefore, we decided to undertake a meta-analysis and trial sequential analysis (TSA) to compare the efficacy and safety of finerenone with that of placebo in patients with CKD. We present the following article in accordance with the PRISMA reporting checklist (available at https://dx.doi. org/10.21037/apm-21-763) (19).

\section{Methods}

\section{Search strategy and information sources}

Electronic databases, including PubMed, Embase, and the Cochrane Library databases, were searched from database inception to December 2020 using items related to "finerenone" and "BAY 94-8862." Literature search details are shown in Table S1. The search was limited to human subjects, and no language restrictions were applied. An additional search was conducted by manually reviewing conference proceedings and the references of review articles if necessary.

\section{Inclusion and exclusion criteria}

The inclusion criteria were as follows: (I) study design: RCT; (II) comparison: evaluating the efficacy and safety of finerenone with that of placebo; (III) population: conducted on the patients with CKD; and (IV) outcome: assessed at least one of the following outcomes: the change in urinary albumin to creatinine ratio (UACR) from baseline to the end of the study, the change in estimated glomerular filtration rate (eGFR) from baseline to the end of the study (see Table S2 for selected time points), adverse events including cardiovascular disorders (including cardiac disorders and vascular disorders) and hyperkalemia. The exclusion criteria were as follows: (I) studies including patients with heart failure but without CKD or studies including patients with CKD but with data that could not be extracted and analyzed from the published results; and (II) studies such as systemic reviews, comments, case reports, conference abstracts, and editorials.

\section{Study selection and data extraction}

Two reviewers (ZF and CL) independently performed the study selection and data extraction. All disagreements between the two reviewers were resolved by third-party adjudication $(\mathrm{QH})$. A standard form designed according to the Cochrane checklist was used to collect data from 


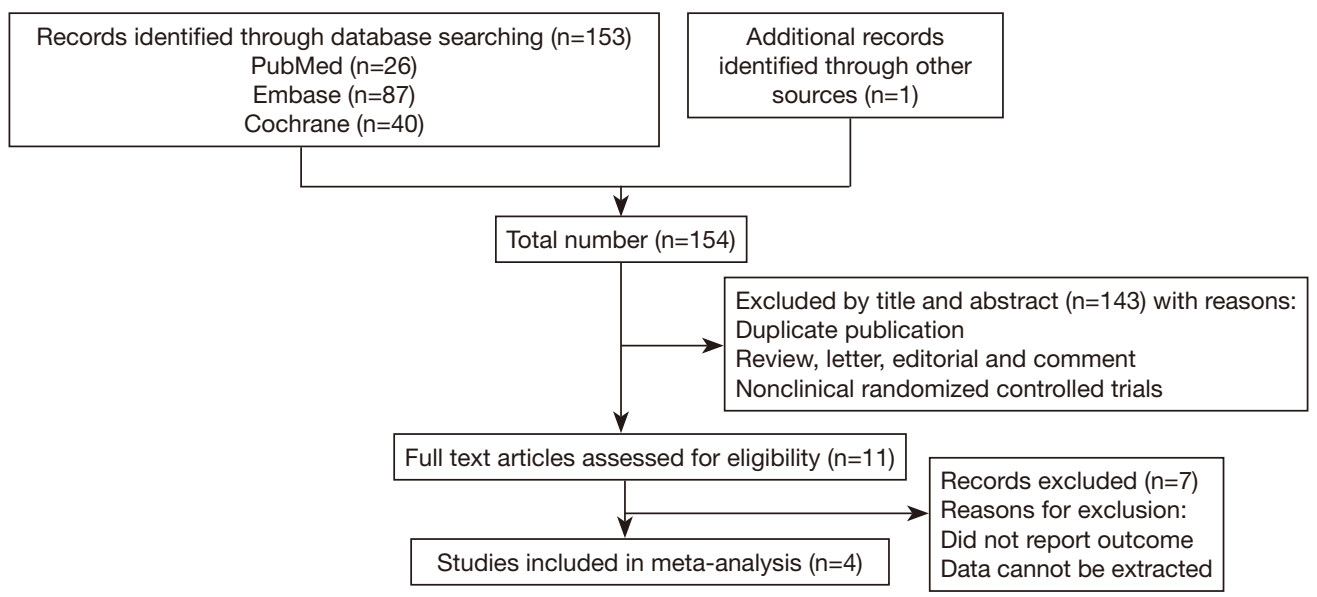

Figure 1 Flow diagram of the study selection process for the meta-analysis.

each study. The extracted data included first author, year of publication, study setting, patient characteristics, sample size, doses of treatment, control, and follow-up.

\section{Assessment of risk of bias}

Assessment of risk of bias was performed by two reviewers (ZF and CL) according to the Cochrane Collaboration's risk-of-bias assessment tool in Review Manager 5.3. Publication bias was assessed by using Stata (version 13.0).

\section{Statistical analysis}

Dichotomous outcomes were reported using relative risks (RRs) with $95 \%$ confidence intervals (CIs), whereas continuous outcomes (UACR and eGFR) were reported using mean differences (MDs) with $95 \%$ CIs. Heterogeneity across the trials was assessed using the $\mathrm{I}^{2}$ statistic, Q statistic and tau-squared test, and $\mathrm{I}^{2}>50 \%$ indicated significant heterogeneity (20). R software (version 3.6.1) was used to calculate the $95 \%$ CIs of these results. The random-effects model was applied to pool the results of all the studies included in this meta-analysis regardless of the heterogeneity, and sensitivity analysis was performed to determine the robustness of the results. Subgroup analysis was conducted to investigate the potential sources of between-study heterogeneity. Publication bias was assessed using Stata with the metabias6 package, version 13.0 (Stata Corporation). A two-tailed $\mathrm{P}$ value less than 0.05 was considered to indicate a statistically significant difference. All statistical analyses were performed using Review Manager, version 5.3 (RevMan, The Cochrane
Collaboration, Oxford, United Kingdom). If the mean or standard deviation of UACR or eGFR could not be directly obtained from the trials, we estimated the mean and deviation from the sample size, median, range and/or interquartile range (21).

\section{Trial sequential analysis}

Trial sequential analysis (TSA) was used in the metaanalyses to reduce the risk of reaching a false-positive or false-negative conclusion (22). When the cumulative $\mathrm{Z}$-curve crossed the trial sequential monitoring boundary or entered the futility area, a sufficient level of evidence for the anticipated intervention effect was reached, and no further trials were needed. If the $Z$-curve did not cross any of the boundaries and the required information size (RIS) had not been reached, evidence was insufficient to obtain a conclusion, and more trials were needed to confirm the results (23). In this TSA, we estimated the RIS based on an RR reduction of $10 \%$. The type I error $(\alpha)$ was maintained at 0.05 (two-sided) in this TSA. We used a power $(1-\beta)$ of 0.90 to calculate the required information size for all the results. The control event proportion was calculated based on the comparator group (24). The TSA was conducted using TSA Version 0.9.5.10 Beta (www.ctu.dk/tsa).

\section{Results}

\section{Characteristics of eligible studies and quality assessment}

The study selection process is depicted as a flow diagram in Figure 1. After the removal of duplicates and studies 
that failed to meet the inclusion criteria, 11 articles were retrieved for detailed assessment. Seven articles were excluded because they did not report outcomes or the data could not be extracted from the articles. In total, 4 RCTs (25-28) involving 7,048 participants were included in this meta-analysis. The characteristics of the included trials and patient demographic data are summarized in Table 1. Three studies $(25,27,28)$ were phase 2 clinical trials, and the other study (26) was a phase 3 clinical trial. These trials were reported between 2013 and 2020. The number of patients included in each study ranged from 96 to 5,674, and the follow-up ranged from 1 to 30 months. Most of the patients involved in these trials received concomitant medication at baseline, including angiotensin-converting enzyme inhibitors (ACEIs), angiotensin receptor blockers (ARBs) and drugs used for diabetes treatment. More patients were taking ARBs than ACEIs. The proportions of patients receiving ACEIs at baseline in the studies conducted by Bakris et al. [2015] (25), Bakris et al. [2020] (26) and Katayama et al. [2017] (27) were $45.7 \%, 34.2 \%$ and 9.4\%, respectively. The proportions of patients receiving ARBs in the studies conducted by Bakris et al. [2015] (25), Bakris et al. [2020] (26) and Katayama et al. [2017] (27) were $55.1 \%, 65.7 \%$ and $90.6 \%$, respectively. Pitt et al. also described the number of patients who were receiving agents acting on the renin-angiotensin system (RAS), and the proportion of these patients was $95.4 \%$ (28). In addition, in the three studies (25-27) dealing with patients with type 2 diabetes and CKD, nearly all the patients (over 95 percent) were receiving glucose-lowering therapies. Review Manager 5.3 was used to evaluate the overall quality of the articles. The quality of the studies is shown in Figure S1. All studies had low selection bias.

\section{Efficacy outcomes}

\section{Urine albumin-to-creatinine ratio (UACR)}

UACR is an important indicator of treatment efficacy and is closely related to the improvement and prognosis of CKD. Three studies $(\mathrm{n}=329)(25,27,28)$ displayed a difference in the urine albumin-to-creatinine ratio between the finerenone and placebo groups. A significantly greater reduction in the urine albumin-to-creatinine ratio among patients with CKD was observed in the finerenone group than in the placebo group [MD, -0.30 (95\% CI, -0.50 to -0.11 ), $\mathrm{P}=0.003, \mathrm{I}^{2}=0 \%$ (95\% CI, $0.0 \%$ to $40.6 \%$ ), $\mathrm{Chi}^{2}=0.03$, $\mathrm{P}=0.98, \mathrm{tau}^{2}=0$ (95\% CI, 0 to 0.17); Figure $\left.2 A\right]$, and TSA confirmed this result (the cumulative $\mathrm{Z}$-curve crossed the conventional boundary and the trial sequential monitoring boundary; Figure 2B). In addition, we compared the clinical effectiveness of finerenone at doses of 10 and $20 \mathrm{mg}$ once daily. The pooled analysis showed that doses of both 10 and $20 \mathrm{mg}$ once daily appear to be efficacious at reducing the UACR [MD, -0.24 (95\% CI, -0.46 to -0.01$), \mathrm{P}=0.04, \mathrm{I}^{2}$ $=0 \%$ and $\mathrm{MD},-0.35$ (95\% CI, -0.57 to -0.13$), \mathrm{P}=0.002, \mathrm{I}^{2}$ $=0 \%$, respectively; Figure $3 A, B]$. However, the TSA result only suggested the treatment effect of the finerenone dose of $20 \mathrm{mg} / \mathrm{d}$ on reducing UACR, for the cumulative $Z$ curve of the dose of $10 \mathrm{mg} / \mathrm{d}$ just crossed the conventional boundary but did not cross the trial sequential monitoring boundary (Figure $3 C$ ) and the cumulative $\mathrm{Z}$ curve of the dose of $20 \mathrm{mg} / \mathrm{d}$ crossed the conventional boundary and the sequential monitoring boundary (Figure 3D).

\section{Estimated glomerular filtration rate (eGFR)}

Three studies $(n=5,974)(25,26,28)$ reported a change in eGFR in patients with CKD. The results demonstrated that there was no significant difference in eGFR between the finerenone group and the placebo group [MD, -0.90 ( $95 \%$ CI, -3.84 to 2.04$), \mathrm{P}=0.55]$. Heterogeneity was observed among the included studies $\left[\mathrm{I}^{2}=86 \%\right.$ (95\% CI, $68.5 \%$ to 95.9\%), $\mathrm{Chi}^{2}=13.89, \mathrm{P}=0.0001, \mathrm{tau}^{2}=0.08$ (95\% CI, 0.01 to 3.54)] (Table 2). Therefore, we applied a sensitivity analysis to further investigate the eGFR results. We found that no between-study variance was observed, and the eGFR results were significantly changed (a significant difference was found in eGFR between the finerenone group and the placebo group) if one study was excluded from the analysis [MD, -2.32 (95\% CI, -4.18 to -0.45$), \mathrm{P}=0.01, \mathrm{I}^{2}=0 \%$, $\mathrm{Chi}^{2}$ $\left.=0.10, \mathrm{P}=0.76, \operatorname{tau}^{2}=0\right](26)$. However, due to the limited number of included studies, further research is needed to achieve a more reliable result.

\section{Adverse events}

Four studies $(n=6,039)(25-28)$ showed that the overall frequency of adverse events was similar between the finerenone and placebo groups [RR, 1.00 (95\% CI, 0.98, 1.02), $\mathrm{P}=0.84, \mathrm{I}^{2}=0 \%$ (95\% CI, $0.0 \%$ to $5.9 \%$ ), $\mathrm{Chi}^{2}=0.47$, $\mathrm{P}=0.93, \operatorname{tau}^{2}=0$ (95\% CI, 0 to 0.05$)$; Figure $4 A$ ], and TSA confirmed this result (the cumulative $Z$ curve crossed the futility boundary and reached the required information size, Figure $4 B$ ). In addition, we compared the incidence of total adverse events at doses of 10 and $20 \mathrm{mg}$ once daily separately. No significant differences were observed between the finerenone and placebo groups in either the 10 or $20 \mathrm{mg} / \mathrm{d}$ 


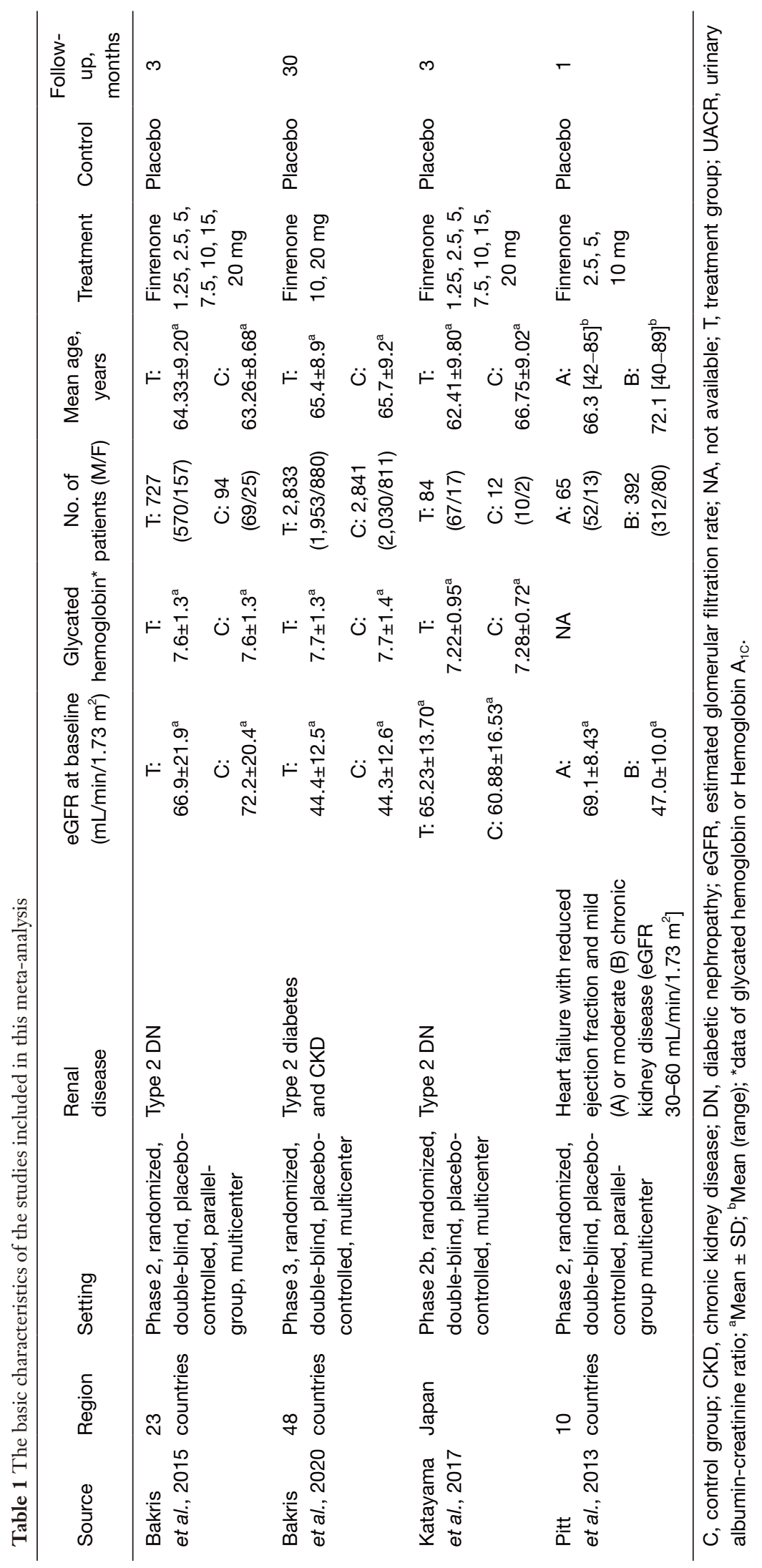




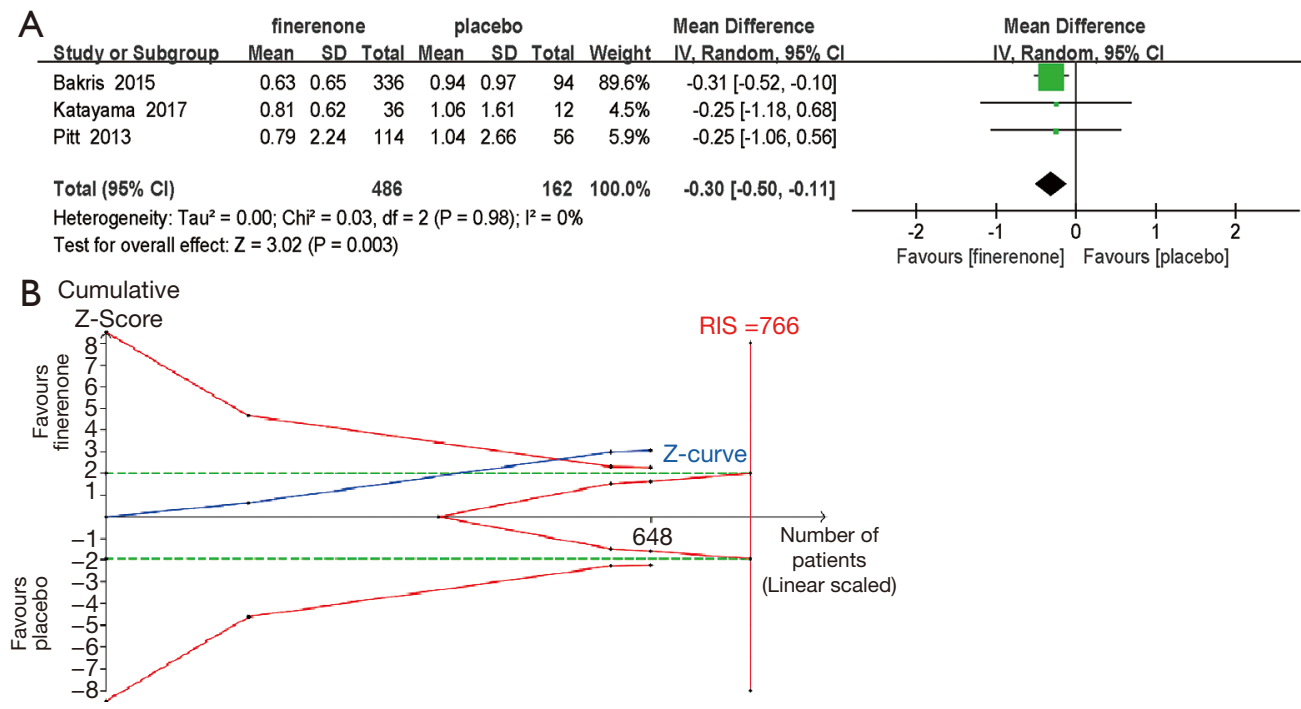

Figure 2 The efficacy outcomes of finerenone vs. placebo. (A) Forest plot of the mean UACR (urine albumin-to-creatinine ratio) to that at baseline. (B) Random effects model of the TSA of UACR. A diversity-adjusted information size of 766 participants was calculated on the basis of using $\alpha=5 \%$ (two-sided), $\beta=10 \%$ (power $90 \%$ ), and $\mathrm{I}^{2}=0 \%$. The solid blue line represents the cumulative Z-curve, which crossed the conventional boundary (dashed green line) and the trial sequential monitoring boundary (solid red line). Data were analyzed using information on doses of at least $10 \mathrm{mg} / \mathrm{d}$.

groups (Table 2). However, the risk of cardiovascular disorders and hyperkalemia related to finerenone was different between the finerenone and placebo groups (Figure 5).

\section{Cardiovascular disorders}

Four trials $(n=6,039)(25-28)$ investigated the incidence of cardiovascular disorders in the finerenone and placebo groups. In patients with CKD, a lower risk of cardiovascular disorders was found in the finerenone group than in the placebo group [RR, 0.92 (95\% CI, 0.85, 0.99), $\mathrm{P}=0.03, \mathrm{I}^{2}$ $=0 \%$; Figure $5 A$, but TSA could not confirm this result (the cumulative $\mathrm{Z}$-curve just crossed the conventional boundary but did not cross the trial sequential monitoring boundary; Figure 5C).

\section{Hyperkalemia}

Four studies $(n=6,039)(25-28)$ indicated a difference in the relative risk of hyperkalemia between the finerenone and placebo groups. The use of finerenone was associated with a higher relative risk of hyperkalaemia than placebo in patients with CKD [RR, 2.04 (95\% CI, 1.77, 2.34), $\mathrm{P}<0.00001, \mathrm{I}^{2}=0 \%$; Figure $\left.5 \mathrm{~B}\right]$, and TSA suggested that the evidence to reach this conclusion was sufficient, as the cumulative $\mathrm{Z}$-curve crossed both the conventional boundary and the trial sequential monitoring boundary (Figure $5 D$ ).

\section{Publication bias}

No potential publication bias was observed in the outcomes of adverse events and UACR $(\mathrm{P}=0.497$ and $\mathrm{P}=0.602$ for Begg's test, $\mathrm{P}=0.924$ and $\mathrm{P}=0.463$ for Egger's test, respectively, see Figures S2,S3) among the included studies, but publication bias was detected in the efficacy outcomes of eGFR ( $\mathrm{P}=0.602$ for Begg's test and $\mathrm{P}=0.041$ for Egger's test, see Figure S4). However, due to the limited number of included trials, the actual publication bias may not be able to be observed via this method. Although publication bias was not detected in the outcomes of adverse events and UACR through Begg's test and Egger's test, the funnel plots of these two outcomes were apparently asymmetric, which indicated that publication bias may actually exist in this meta-analysis. Therefore, further research is needed to assess the potential publication bias more accurately and achieve a more reliable conclusion.

\section{Discussion}

Finerenone (BAY 94-8862), a novel nonsteroidal, selective mineralocorticoid receptor antagonist (MRA), is the third generation of MRAs. Finerenone has a better selectivity than spironolactone and a stronger MR-binding affinity 

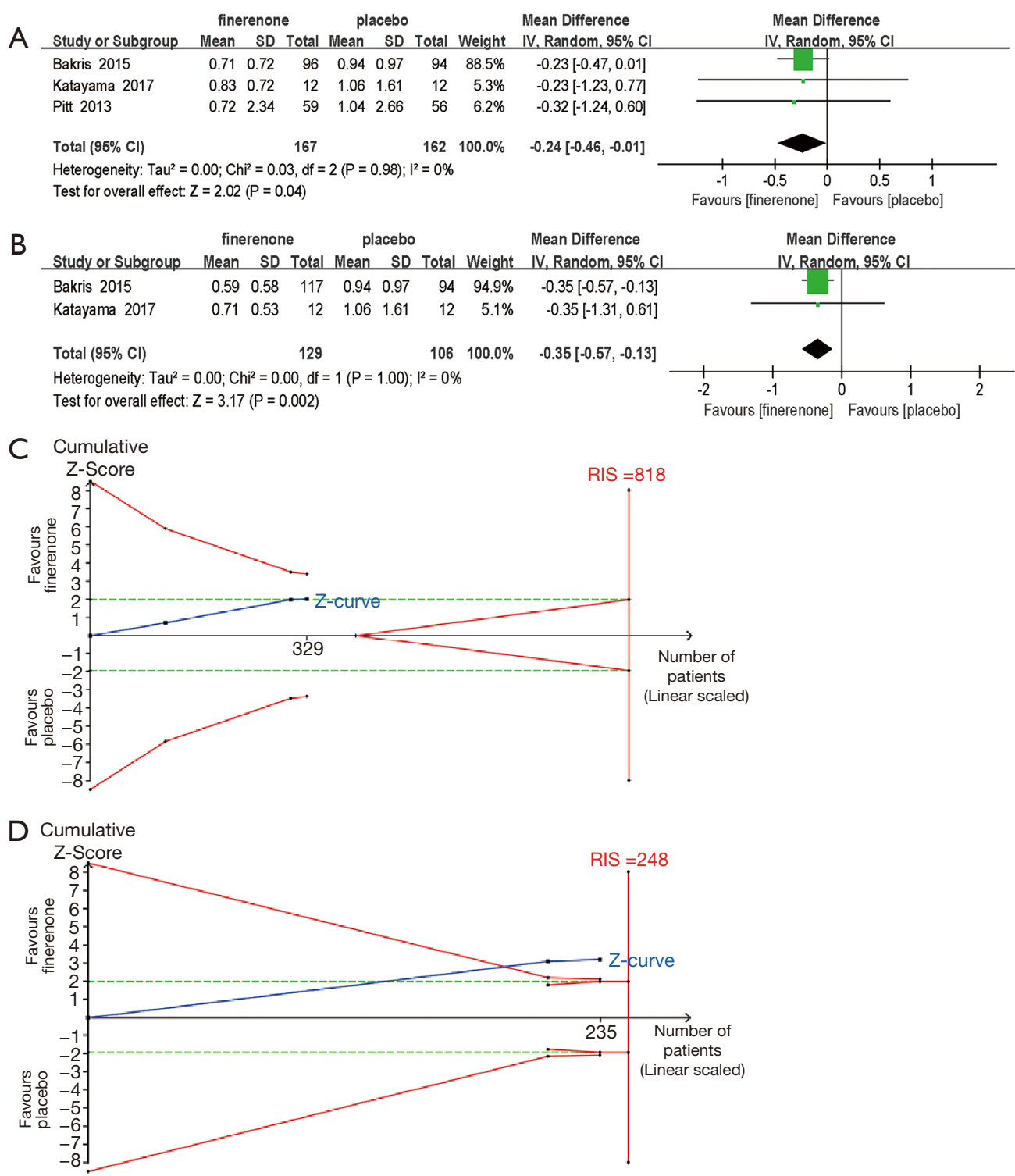

Figure 3 The UACR results for different doses. (A) Forest plot comparing the mean UACR (urine albumin-to-creatinine ratio) to that at baseline in the $10 \mathrm{mg}$ group. (B) Forest plot of the UACR in the $20 \mathrm{mg}$ group. (C) TSA for $10 \mathrm{mg}$ finerenone. A diversity-adjusted information size of 818 participants was calculated on the basis of using $\alpha=5 \%$ (two-sided), $\beta=10 \%$ (power $90 \%$ ), and $\mathrm{I}^{2}=0 \%$. The solid blue line represents the cumulative Z-curve, which crossed the conventional boundary (dashed green line) but did not cross the trial sequential monitoring boundary (solid red line). (D) TSA for $20 \mathrm{mg}$ finerenone. A diversity-adjusted information size of 248 participants was calculated on the basis of using $\alpha=5 \%$ (two-sided), $\beta=10 \%$ (power $90 \%$ ), and $\mathrm{I}^{2}=0 \%$. The solid blue line represents the cumulative Z-curve, which crossed the conventional boundary (dashed green line) and the trial sequential monitoring boundary (solid red line).

than eplerenone (classic steroidal MRAs) $(17,18)$. In this meta-analysis, we used a dose of at least $10 \mathrm{mg} / \mathrm{d}$ to assess the overall efficacy and safety of finerenone for its best potential effect $(29,30)$ and compared the outcomes of UACR and total adverse events at doses of 10 and $20 \mathrm{mg}$ of finerenone separately. In addition, we further used TSA to provide more conservative estimates and to establish more sufficient and conclusive evidence of the outcomes.

Previous meta-analyses have not yet evaluated this topic since finerenone is a relatively new drug. In this updated 
Table 2 Comparison of the other outcomes of finerenone

\begin{tabular}{llllll}
\hline Outcomes & Dose & Number of studies & Mean difference $(95 \% \mathrm{Cl})$ & Test for effect $(\mathrm{P}$ value $)$ & ${\text { Heterogeneity } \mathrm{I}^{2}(\mathrm{P} \text { value })}$ \\
\hline eGFR & $\geq 10 \mathrm{mg}$ q.d. & $3(22,23,25)$ & $-0.90(-3.84,2.04)$ & 0.55 & $86 \%(0.001)$ \\
Adverse events & $10 \mathrm{mg}$ q.d. & $3(22,24,25)$ & $1.09(0.89,1.32)$ & 0.41 & $0 \%(0.61)$ \\
Adverse events & $20 \mathrm{mg}$ q.d. & $2(22,24)$ & $1.05(0.82,1.35)$ & 0.68 & $0 \%(0.58)$ \\
\hline
\end{tabular}

$\mathrm{Cl}$, confidence interval; eGFR, estimated glomerular filtration rate, $\left(\mathrm{mL} / \mathrm{min} / 1.73 \mathrm{~m}^{2}\right)$; q.d., once daily; UACR, urine albumin-to-creatinine ratio.

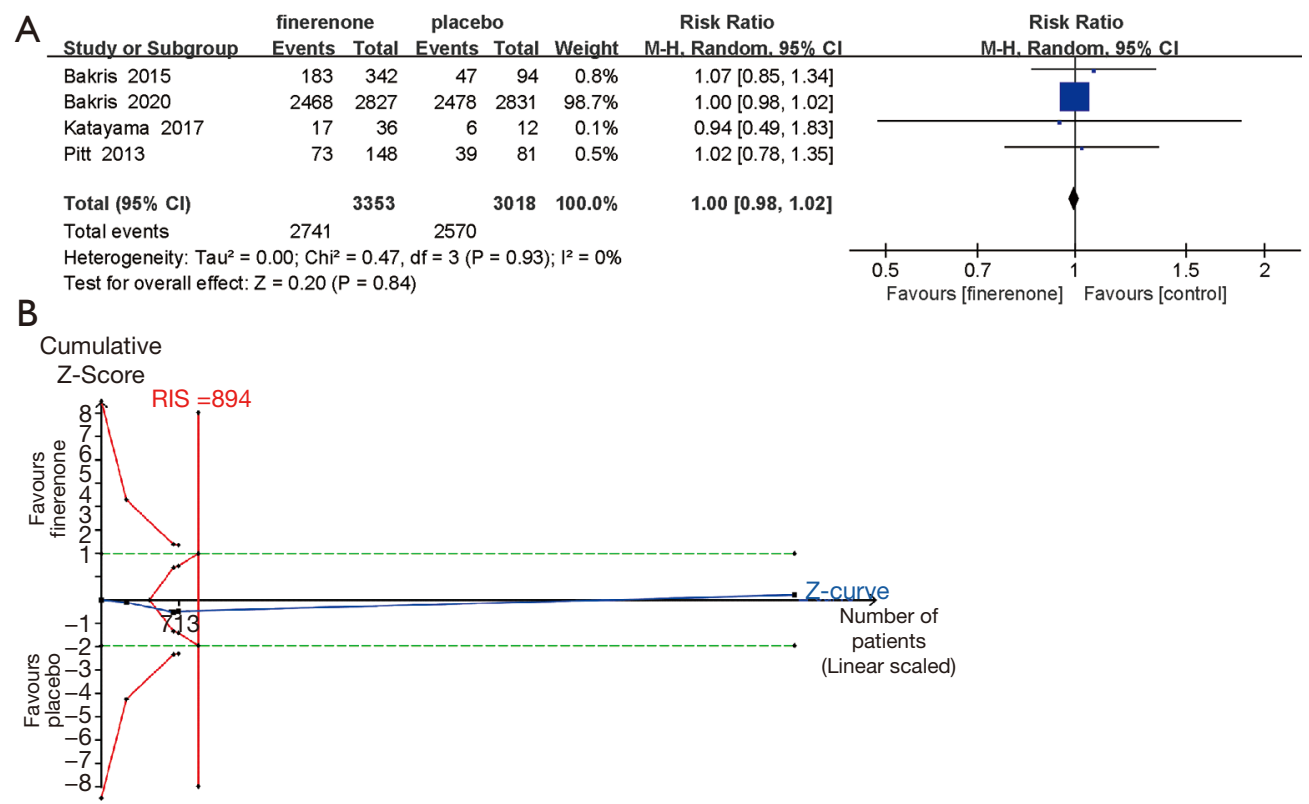

Figure 4 The incidence of adverse events in the finerenone and placebo groups. (A) Forest plot of the adverse events. (B) Random effects model of the TSA of adverse events. A diversity-adjusted information size of 894 participants was calculated on the basis of using $\alpha=5 \%$ (two-sided), $\beta=10 \%$ (power $90 \%$ ), and $\mathrm{I}^{2}=0 \%$. The solid blue line represents the cumulative Z-curve, which crossed the futility boundary (solid red line).

meta-analysis with the largest sample size to date, we found (I) there was a significant decrease in UACR in the finerenone treatment groups compared with the UACR in the placebo group in patients with CKD, and TSA confirmed this result; (II) doses of both 10 and $20 \mathrm{mg}$ once daily seem to be efficacious at reducing UACR, and TSA confirmed the effectiveness of $20 \mathrm{mg}$, with no increase in the risk of adverse events; (III) there were no statistically significant changes in eGFR between the two groups; (IV) the incidence of adverse events was similar in the two groups in patients with CKD, and TSA suggested this conclusion was reliable; (V) compared with placebo, finerenone greatly reduced the risk of cardiovascular disorders in CKD patients, but TSA did not prove this result; and (VI) more episodes of hyperkalemia were observed in the finerenone groups than the placebo groups, and TSA could confirm this result.

As albuminuria is a significant predictor of the progression of CKD (31), a decrease in albuminuria is closely related to the protection of renal function. This meta-analysis suggested that compared with placebo, the use of finerenone may have a great advantage in reducing UACR in patients with CKD. The rationale of this approach is that excess aldosterone induces renal and cardiovascular injury because of its proinflammatory and profibrotic effects (32-34). Thus, blockade of aldosterone could be effective for these patients.

However, analysis of the three studies $(25,26,28)$ comparing finerenone to placebo revealed no significant 


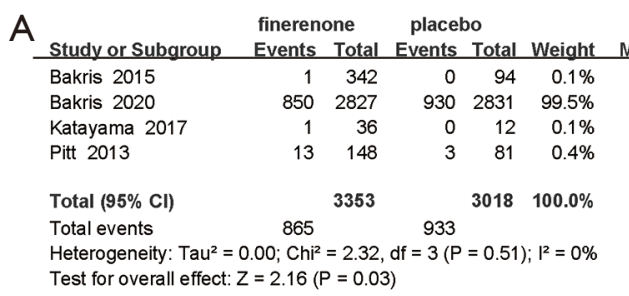

Risk Ratio Risk Ratio

M-H. Random. $95 \% \mathrm{Cl}$ M-H. Random. $95 \% \mathrm{Cl}$

$0.83[0.03,20.23]$

$0.92[0.85,0.99]$

$1.05[0.05,24.29]$

$2.37[0.70,8.08]$

$0.92[0.85,0.99]$

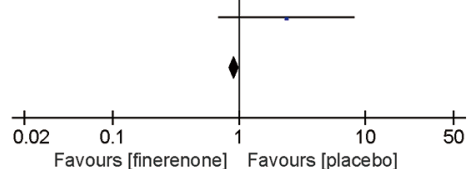

B

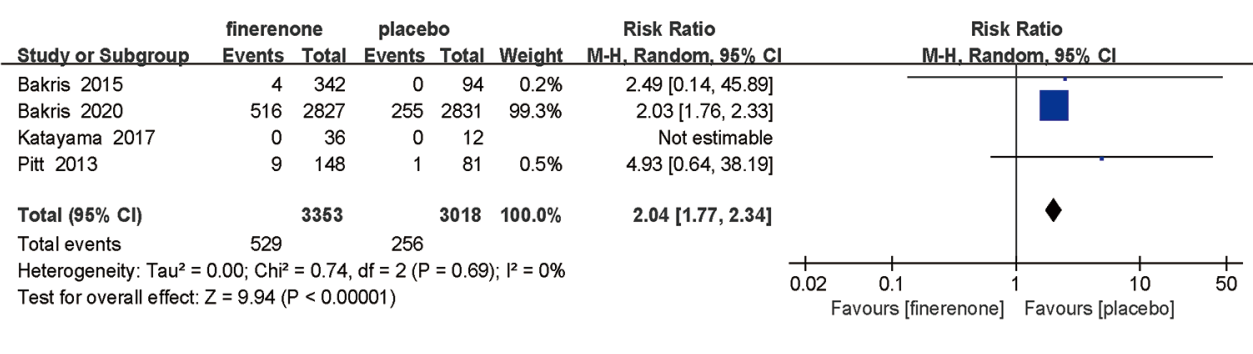

C

Cumulative
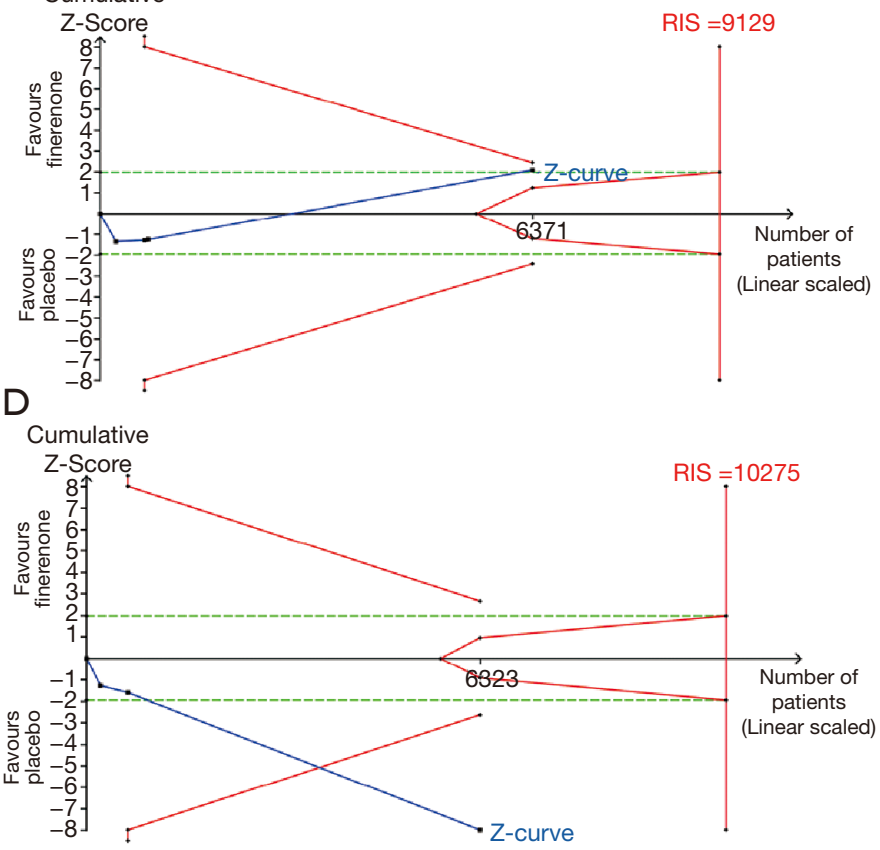

Figure 5 The incidence of cardiovascular disorders and hyperkalemia in the finerenone and placebo groups. (A) Forest plot of cardiovascular disorders. (B) Forest plot of hyperkalemia. (C) Random effects model of the TSA of cardiovascular disorders. A diversityadjusted information size of 9,129 participants was calculated on the basis of using $\alpha=5 \%$ (two-sided), $\beta=10 \%$ (power $90 \%$ ), and $\mathrm{I}^{2}=0 \%$. The solid blue line represents the cumulative Z-curve, which crossed the conventional boundary (dashed green line) but did not cross the trial sequential monitoring boundary (solid red line). (D) Random effects model of the TSA of hyperkalemia. A diversity-adjusted information size of 10,275 participants was calculated on the basis of using $\alpha=5 \%$ (two-sided), $\beta=10 \%$ (power $90 \%$ ), and $\mathrm{I}^{2}=0 \%$. The solid blue line represents the cumulative Z-curve, which crossed the conventional boundary (dashed green line) and the trial sequential monitoring boundary (solid red line). 
changes in eGFR between the two groups. Nevertheless, the change in eGFR in the finerenone group was much different from that in the placebo group and is directly related to the follow-up time (26). A study showed a smaller decrease in eGFR in the finerenone group than in the placebo group in the observation after 12 months, and the trend persisted throughout the trial (26). This result indicates that finerenone may have a long-term benefit for patients with renal diseases. This finding may be of great significance since the patients for whom this treatment might be suitable all have chronic disease and require longterm medication. Therefore, further studies investigating eGFR changes in different periods are needed to assess this outcome.

Kidney failure, which is defined as end-stage renal disease (ESRD) or a sustained eGFR of $<15 \mathrm{~mL}$ per minute per $1.73 \mathrm{~m}^{2}$ of body-surface area (26), is also a key outcome worth assessing in CKD patients since CKD will finally progress to renal failure without appropriate treatment. One trial (26) included in this meta-analysis reported that the incidence of kidney failure was significantly lower in the finerenone group than in the placebo group. This indicates that finerenone may have an effect on ameliorating the progression of CKD and thus benefit patients with CKD.

A major concern of the use of finerenone is its adverse events. Two main adverse events were reported in this study. Compared with placebo, finerenone had less unfavorable effects on cardiovascular disorders in patients with CKD, but TSA could not confirm this result. Thus, more trials are needed to make a more reliable conclusion. In terms of hyperkalemia, the pooled result indicated that compared with placebo, the use of finerenone led to an increased risk of hyperkalemia in patients with CKD, and TSA confirmed this result. This may be associated with the treatment mechanism of MRAs. MRA use increases sodium excretion and decreases potassium excretion in the kidney, resulting in an increase in serum potassium levels (35). Even though the incidence of hyperkalaemia was increased in comparison with that in the placebo group, finerenone is associated with a lower risk than classic steroidal MRAs (spironolactone or eplerenone) according to recent trials and meta-analyses $(30,35,36)$. Current research has reported that compared with eplerenone, finerenone has a much better effect on preventing cardiac fibrosis and improving strain parameters in mice (37). Hence, future research on finerenone is necessary to explore its efficacy and safety versus that of spironolactone and/or eplerenone.

In addition, patients with CKD usually receive a combination of medications including an antidiabetic prescription medication and/or antihypertensive drugs such as angiotensin-converting enzyme inhibitors (ACEIs) or angiotensin receptor blockers (ARBs). Therefore, it is necessary to consider how the effects of finerenone might vary according to the use of other medications. Current studies have revealed that dual renin-angiotensinaldosterone system (RAAS) blockade with ACEI plus ARB or $\mathrm{ARB}$ plus renin inhibition is associated with an increased risk of serious adverse events such as acute kidney injury or stroke, and no apparent benefits are seen in the context of this kind of medication (38). In contrast, finerenone added to a single RAS blockade seems to be associated with greater reductions in urine albumin or protein excretion than placebo or dual RAS blockade along with fewer episodes of hyperkalaemia (39).

\section{Limitations}

Our analysis has some limitations. First, the number of studies included in this analysis was small, and the data for some of the results were scarce. Second, this article only provides preliminary results on the efficacy and safety of finerenone. Third, most of the studies included in the present analysis had a short follow-up period, and only one study had a planned follow-up of 65 months. Thus, further evidence and conclusive results regarding the shortterm and long-term efficacy and safety of finerenone in comparison with spironolactone or eplerenone are expected.

\section{Conclusions}

This analysis suggests that finerenone confers an important antiproteinuric effect on patients with CKD, with a less unfavorable effect on eGFR. Although a higher risk of hyperkalemia was observed for finerenone than for placebo, the use of finerenone results in a lower incidence of cardiovascular disorders in patients with CKD. Finerenone seems to be a new kind of promising treatment for patients with CKD.

\section{Acknowledgments}

Funding: This work was supported by grants from the Fostering Fund of Chinese PLA General Hospital for National Distinguished Young Scholar Science Fund (2019-JQPY-002), National Natural Science Foundation of China (Nos. 81870491 and 82070741) and the National 
Key Research and Development Project of China (2018YFE0126600).

\section{Footnote}

Reporting Checklist: The authors have completed the PRISMA reporting checklist. Available at https://dx.doi. org/10.21037/apm-21-763

Peer Review File: Available at https://dx.doi.org/10.21037/ apm-21-763

Conflicts of Interest: All authors have completed the ICMJE uniform disclosure form (available at https://dx.doi. org/10.21037/apm-21-763). The authors have no conflicts of interest to declare.

Ethical Statement: The authors are accountable for all aspects of the work in ensuring that questions related to the accuracy or integrity of any part of the work are appropriately investigated and resolved.

Open Access Statement: This is an Open Access article distributed in accordance with the Creative Commons Attribution-NonCommercial-NoDerivs 4.0 International License (CC BY-NC-ND 4.0), which permits the noncommercial replication and distribution of the article with the strict proviso that no changes or edits are made and the original work is properly cited (including links to both the formal publication through the relevant DOI and the license). See: https://creativecommons.org/licenses/by-nc-nd/4.0/.

\section{References}

1. Muskiet MHA, Wheeler DC, Heerspink HJL. New pharmacological strategies for protecting kidney function in type 2 diabetes. Lancet Diabetes Endocrinol 2019;7:397-412.

2. Sloan LA. Review of glucagon-like peptide-1 receptor agonists for the treatment of type 2 diabetes mellitus in patients with chronic kidney disease and their renal effects. J Diabetes 2019;11:938-48.

3. Thomas MC, Cooper ME, Zimmet P. Changing epidemiology of type 2 diabetes mellitus and associated chronic kidney disease. Nat Rev Nephrol 2016;12:73-81.

4. Hill NR, Fatoba ST, Oke JL, et al. Global Prevalence of Chronic Kidney Disease - A Systematic Review and MetaAnalysis. PLoS One 2016;11:e158765.
5. Ingelfinger JR, Rosen CJ. Finerenone - Halting Relative Hyperaldosteronism in Chronic Kidney Disease. N Engl J Med 2020;383:2285-6.

6. Gilbert KC, Brown NJ. Aldosterone and inflammation. Curr Opin Endocrinol Diabetes Obes 2010;17:199-204.

7. Bianchi S, Bigazzi R, Campese VM. Long-term effects of spironolactone on proteinuria and kidney function in patients with chronic kidney disease. Kidney Int 2006;70:2116-23.

8. Boesby L, Elung-Jensen T, Klausen TW, et al. Moderate antiproteinuric effect of add-on aldosterone blockade with eplerenone in non-diabetic chronic kidney disease. A randomized cross-over study. PLoS One 2011;6:e26904.

9. Lin YC, Lin JW, Wu MS, et al. Effects of calcium channel blockers comparing to angiotensin-converting enzyme inhibitors and angiotensin receptor blockers in patients with hypertension and chronic kidney disease stage 3 to 5 and dialysis: A systematic review and meta-analysis. PLoS One 2017;12:e0188975.

10. Currie G, Taylor AH, Fujita T, et al. Effect of mineralocorticoid receptor antagonists on proteinuria and progression of chronic kidney disease: a systematic review and meta-analysis. BMC Nephrol 2016;17:127.

11. Epstein M, Williams GH, Weinberger M, et al. Selective aldosterone blockade with eplerenone reduces albuminuria in patients with type 2 diabetes. Clin J Am Soc Nephrol 2006;1:940-51.

12. Weir MR, Lakkis JI, Jaar B, et al. Use of ReninAngiotensin System Blockade in Advanced CKD: An NKF-KDOQI Controversies Report. Am J Kidney Dis 2018;72:873-84.

13. Navaneethan SD, Nigwekar SU, Sehgal AR, et al. Aldosterone antagonists for preventing the progression of chronic kidney disease: a systematic review and metaanalysis. Clin J Am Soc Nephrol 2009;4:542-51.

14. Schjoedt KJ, Rossing K, Juhl TR, et al. Beneficial impact of spironolactone in diabetic nephropathy. Kidney Int 2005;68:2829-36.

15. Schjoedt KJ, Rossing K, Juhl TR, et al. Beneficial impact of spironolactone on nephrotic range albuminuria in diabetic nephropathy. Kidney Int 2006;70:536-42.

16. Sun LJ, Sun YN, Shan JP, et al. Effects of mineralocorticoid receptor antagonists on the progression of diabetic nephropathy. J Diabetes Investig 2017;8:609-18.

17. Bramlage P, Swift SL, Thoenes M, et al. Non-steroidal mineralocorticoid receptor antagonism for the treatment of cardiovascular and renal disease. Eur J Heart Fail 2017;19:811. 
18. Kolkhof P, Delbeck M, Kretschmer A, et al. Finerenone, a novel selective nonsteroidal mineralocorticoid receptor antagonist protects from rat cardiorenal injury. J Cardiovasc Pharmacol 2014;64:69-78.

19. Moher D, Liberati A, Tetzlaff J, et al. Preferred reporting items for systematic reviews and meta-analyses: the PRISMA statement. PLoS Med 2009;6:e1000097.

20. Higgins JP, Thompson SG, Deeks JJ, et al. Measuring inconsistency in meta-analyses. BMJ 2003;327:557-60.

21. Wan X, Wang W, Liu J, et al. Estimating the sample mean and standard deviation from the sample size, median, range and/or interquartile range. BMC Med Res Methodol 2014;14:135.

22. Brok J, Thorlund K, Gluud C, et al. Trial sequential analysis reveals insufficient information size and potentially false positive results in many meta-analyses. J Clin Epidemiol 2008;61:763-9.

23. Liu C, Mao Z, Kang H, et al. Regional citrate versus heparin anticoagulation for continuous renal replacement therapy in critically ill patients: a meta-analysis with trial sequential analysis of randomized controlled trials. Crit Care 2016;20:144.

24. Liu C, Lu G, Wang D, et al. Balanced crystalloids versus normal saline for fluid resuscitation in critically ill patients: A systematic review and meta-analysis with trial sequential analysis. Am J Emerg Med 2019;37:2072-8.

25. Bakris GL, Agarwal R, Chan JC, et al. Effect of Finerenone on Albuminuria in Patients With Diabetic Nephropathy: A Randomized Clinical Trial. JAMA 2015;314:884-94.

26. Bakris GL, Agarwal R, Anker SD, et al. Effect of Finerenone on Chronic Kidney Disease Outcomes in Type 2 Diabetes. N Engl J Med 2020;383:2219-29.

27. Katayama S, Yamada D, Nakayama M, et al. A randomized controlled study of finerenone versus placebo in Japanese patients with type 2 diabetes mellitus and diabetic nephropathy. J Diabetes Complications 2017;31:758-65.

28. Pitt B, Kober L, Ponikowski P, et al. Safety and tolerability of the novel non-steroidal mineralocorticoid receptor antagonist BAY 94-8862 in patients with chronic heart failure and mild or moderate chronic kidney disease: a randomized, double-blind trial. Eur Heart J 2013;34:2453-63.

29. Snelder N, Heinig R, Drenth HJ, et al. Population Pharmacokinetic and Exposure-Response Analysis of Finerenone: Insights Based on Phase IIb Data and Simulations to Support Dose Selection for Pivotal Trials in Type 2 Diabetes with Chronic Kidney Disease. Clin Pharmacokinet 2020;59:359-70.

30. Pei H, Wang W, Zhao D, et al. The use of a novel non-steroidal mineralocorticoid receptor antagonist finerenone for the treatment of chronic heart failure: A systematic review and meta-analysis. Medicine (Baltimore) 2018;97:e0254.

31. de Zeeuw D, Remuzzi G, Parving HH, et al. Proteinuria, a target for renoprotection in patients with type 2 diabetic nephropathy: lessons from RENAAL. Kidney Int 2004;65:2309-20.

32. Epstein M. Aldosterone as a mediator of progressive renal disease: pathogenetic and clinical implications. Am J Kidney Dis 2001;37:677-88.

33. Hostetter TH, Rosenberg ME, Ibrahim HN, et al. Aldosterone in progressive renal disease. Semin Nephrol 2001;21:573-9.

34. Waanders F, de Vries LV, van Goor H, et al. Aldosterone, from (patho)physiology to treatment in cardiovascular and renal damage. Curr Vasc Pharmacol 2011;9:594-605.

35. Patel V, Joharapurkar A, Jain M. Role of mineralocorticoid receptor antagonists in kidney diseases. Drug Dev Res 2021;82:341-63.

36. Yang $P$, Shen $W$, Chen $X$, et al. Comparative efficacy and safety of mineralocorticoid receptor antagonists in heart failure: a network meta-analysis of randomized controlled trials. Heart Fail Rev 2019;24:637-46.

37. Grune J, Beyhoff N, Smeir E, et al. Selective Mineralocorticoid Receptor Cofactor Modulation as Molecular Basis for Finerenone's Antifibrotic Activity. Hypertension 2018;71:599-608.

38. Lytvyn Y, Godoy LC, Scholtes RA, et al. Mineralocorticoid Antagonism and Diabetic Kidney Disease. Curr Diab Rep 2019;19:4.

39. Sarafidis PA, Memmos E, Alexandrou ME, et al. Mineralocorticoid Receptor Antagonists for Nephroprotection: Current Evidence and Future Perspectives. Curr Pharm Des 2018;24:5528-36.

Cite this article as: Fu Z, Geng X, Chi K, Song C, Wu D, Liu C, Hong Q. Efficacy and safety of finerenone in patients with chronic kidney disease: a systematic review with meta-analysis and trial sequential analysis. Ann Palliat Med 2021;10(7):74287439. doi: 10.21037/apm-21-763 


\section{Supplementary}

Table S1 The literature search strategy and the results of searching in PubMed

\begin{tabular}{|c|c|c|}
\hline Search step & Query & Results \\
\hline 3 & $\begin{array}{l}\text { ((((((Randomized controlled trial[Publication Type]) OR (controlled clinical trial[Publication Type])) OR } \\
\text { (randomized[Title/Abstract])) OR (placebo[Title/Abstract])) OR (randomly[Title/Abstract])) OR (trial[Title/Abstract])) } \\
\text { OR (groups[Title/Abstract])) NOT ((Animals[MeSH Terms]) NOT (humans[MeSH Terms])) }\end{array}$ & 2783946 \\
\hline 4 & $\begin{array}{l}((((((\text { Chronic kidney disease[MeSH Major Topic]]) OR (chronic kidney disease[Title/Abstract])) OR (chronic renal } \\
\text { failure[Title/Abstract]])) OR (diabetic nephropathy[Title/Abstract]])) OR (diabetic kidney disease[Title/Abstract]])) AND } \\
((\text { finerenone) OR (BAY 94-8862))) AND (((((((Randomized controlled trial[Publication Type]) OR (controlled clinical } \\
\text { trial[Publication Type])) OR (randomized[Title/Abstract])) OR (placebo[Title/Abstract])) OR (randomly[Title/Abstract])) } \\
\text { OR (trial[Title/Abstract])) OR (groups[Title/Abstract]])) NOT ((Animals[MeSH Terms]) NOT (humans[MeSH Terms]))) }\end{array}$ & 26 \\
\hline
\end{tabular}

Generally, electronic databases including PubMed, Embase, and the Cochrane Library databases were searched from database inception to December 2020. The search items used were "chronic kidney disease", "chronic renal failure", "diabetic nephropathy", "diabetes mellitus", and "finerenone", "BAY 94-8862", "randomized controlled trial". The search was limited to human subjects, and no language restrictions were applied. A further search was conducted by manually reviewing conference proceedings and the references of review articles to identify potentially relevant studies. The search terms in PubMed for example, are as follows: (()((Chronic kidney disease[MeSH Major Topic]) OR (chronic kidney disease[Title/Abstract])) OR (chronic renal failure[Title/Abstract])) OR (diabetic nephropathy[Title/Abstract])) OR (diabetic kidney disease[Title/Abstract])) AND ((finerenone) OR (BAY 94-8862))) AND (((((((Randomized controlled trial[Publication Type]) OR (controlled clinical trial[Publication Type])) OR (randomized[Title/Abstract])) OR (placebo[Title/Abstract])) OR (randomly[Title/Abstract])) OR (trial[Title/Abstract])) OR (groups[Title/Abstract])) NOT ((Animals[MeSH Terms]) NOT (humans[MeSH Terms]))).

Table S2 The time points of urine albumin-to-creatinine ratio (UACR) and estimated glomerular filtration rate (eGFR) selected for analysis

\begin{tabular}{lll}
\hline Study & Selected time points of the outcomes & \\
\hline & UACR & eGFR \\
Bakris et al., 2015 & baseline visit and end of study visit (month 3) & baseline visit and end of study visit (month 3) \\
Bakris et al., 2020 & baseline visit and end of study visit (month 30) & baseline visit and end of study visit (month 30) \\
Katayama et al., 2017 & baseline visit and end of study visit (month 3) & baseline visit and end of study visit (month 3) \\
Pitt et al., 2013 & baseline visit and end of study visit (month 1) & baseline visit and end of study visit (month 1) \\
\hline
\end{tabular}

UACR, urinary albumin-creatinine ratio; eGFR, estimated glomerular filtration rate. 

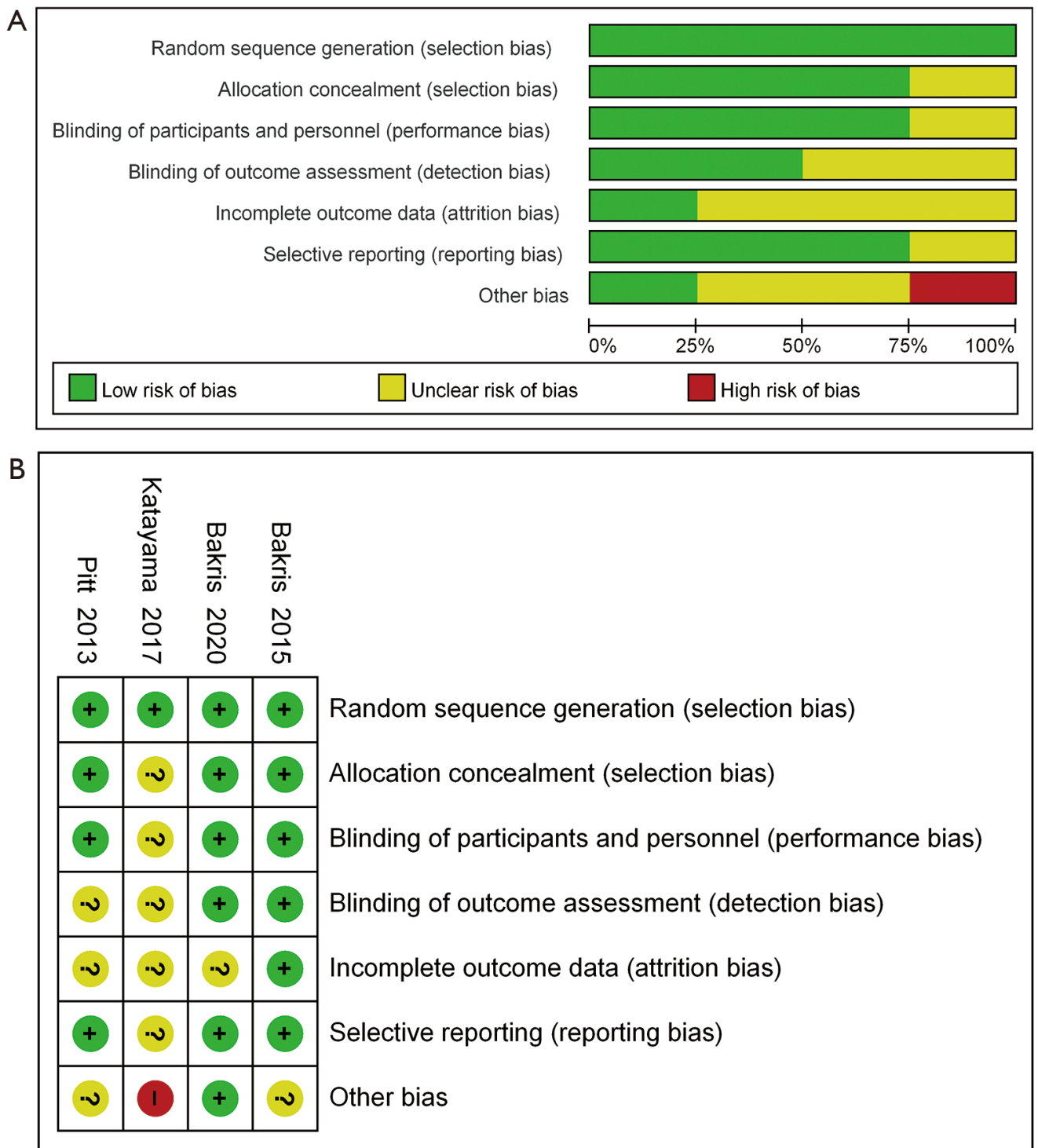

Figure S1 Risk of bias of included studies. (A) Risk of bias graph. (B) Risk of bias summary.
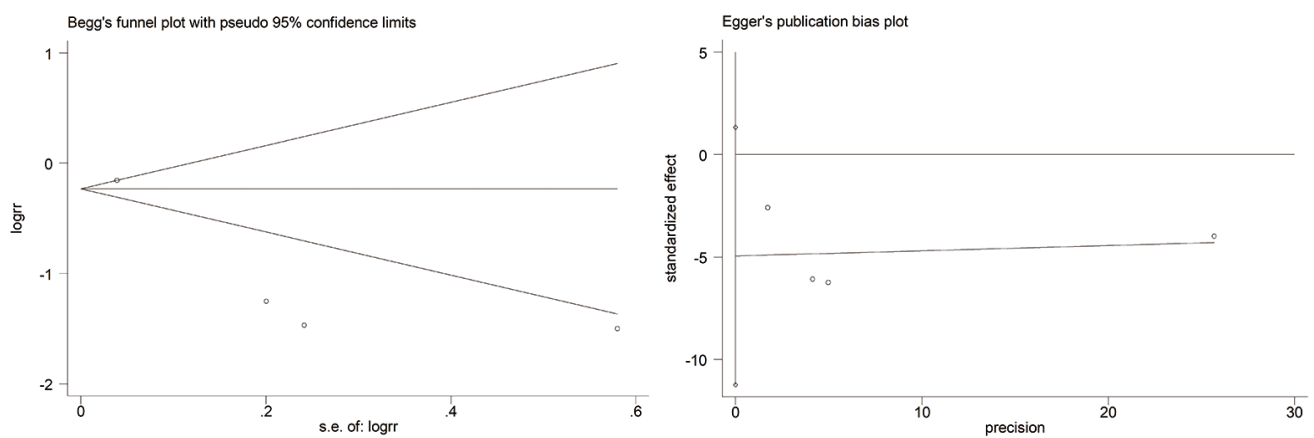

Figure S2 Publication bias of funnel plot for adverse events. 

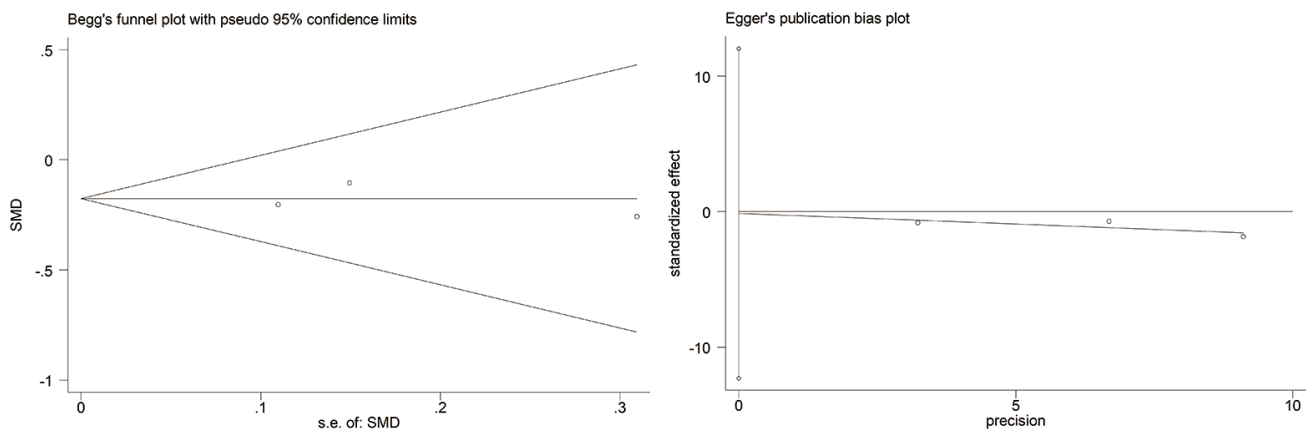

Figure S3 Publication bias of funnel plot for UACR.
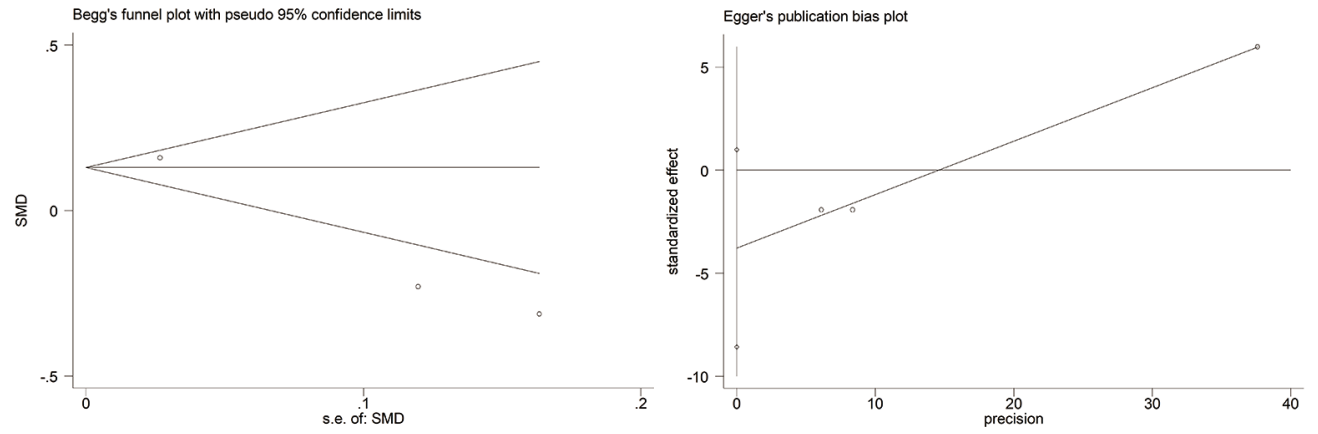

Figure S4 Publication bias of funnel plot for eGFR. 